\section{Stability of early anterior open bite treatment: a 2-year follow- up randomized clinical trial}

Flaviana Alves Dias (D1, Paula Vanessa Pedron Oltramari (D1, Marcio Rodrigues de Almeida (D1, Ana Claúdia de Castro Ferreira Conti (D1, Renato Rodrigues de Almeida (D1, Thais Maria Freire Fernandes (D1.

This 2-year-follow up study compared and evaluated the stability of early anterior open bite $(\mathrm{AOB})$ treatment based on different appliances. Children from 7 to 10 years with Angle Class I, AOB larger than $1.0 \mathrm{~mm}$ and fully erupted maxillary and mandibular permanent central incisors were eligible. The initial sample was 99 patients distributed, by simple randomization, into four groups: BS (bonded spurs), CC (chincup), FPC (fixed palatal crib) and RPC (removable palatal crib). Cephalometric analysis was performed at baseline (T1), final (T2) and 2-year post-treatment (T3) by taking the overbite measurements as the main outcome. Blinding was possible to cephalometric analysis. At T3, with dropouts, there were 63 individuals, being $B S(n=15$; overbite $0.19 \mathrm{~mm} ; 11.54$ years; 10 female $(\mathrm{F}) / 5$ male $(\mathrm{M})) ; \mathrm{CC}(\mathrm{n}=11$; overbite $-0.19 \mathrm{~mm} ; 11.41$ years; $8 \mathrm{~F} / 3 \mathrm{M}) ; \mathrm{FPC}(\mathrm{n}=21$; overbite $1.23 \mathrm{~mm} ; 11.44$ years; $15 \mathrm{~F} / 6 \mathrm{M})$ and; $\mathrm{RPC}(\mathrm{n}=16$; overbite $0.73 \mathrm{~mm} ; 11.67$ years; $6 \mathrm{~F} / 10 \mathrm{M})$. Changes in dentoskeletal variables and breaking deleterious oral habits during the follow up were statically analyzed with $p<.05$. Mandibular skeletal linear measurements and vertical dental components have gradually increased with age, manly at pubertal growth spurt and at the establishment of permanent dentition after treatment. Incisor teeth extrusion had impact on $A O B$ correction and stability in 4 groups, which recorded a $1.15 \mathrm{~mm}$-improvement of overbite after treatment (T3-T2). The experimental appliances were effective with stable results, being FPC the device recorded the highest AOB correction and the lowest patient withdrawal rate.
1 Department of Orthodontics, University of Northern Parana (UNOPAR), Brazil.
Correspondence: Thais Maria Freire Fernandes Department of Orthodontics, University of Northern Parana (UNOPAR), Brazil; 183 Marselha Street - Jd Piza. Londrina, Parana Brazil. Zip Code: 86041-140 / Phone/Fax: $+55(43) 3371-7991$

e-mail: thaismaria@hotmail.com

Key Words: interceptive orthodontics, open bite, cephalometry, follow-up studies

\title{
Introduction
}

Anterior open bite $(\mathrm{AOB})$ is a malocclusion characterized by negative overbite between anterior teeth. It leads to aesthetic, speech, dietary and psychological issues(1) and presents high relapse rates.(2) Its complex and multifactorial etiology encompasses hereditary and respiratory factors, deleterious oral habits, as well as inadequate lingual function and posture.(1) AOB affects approximately $17 \%$ of individuals with mixed dentition;(3) this incidence often increases to $36.3 \%$ when AOB is associated with sucking habits,(3) a fact that significantly requires orthodontic intervention.

Post-treatment stability remains a difficult goal to be achieved $(2,4,5)$ after patients have successfully reached positive overbite during treatment. $(5,6)$ Previous studies have recommended making early intervention in deciduous or mixed dentition, in order to control AOB relapses, restore muscle functions and enable normal facial growth.(7) Clinical studies conducted with children with mixed dentition have shown stability index of 95\%(8) and 96\%,(9) whereas studies conducted with individuals with permanent dentition recorded lower rates, which ranged from $61.3 \%$ to $74.2 \% .(2,4)$

Although several articles(7,10-14) have reported positive results of AOB orthodontic and surgical treatments with significant correction there are limited high-level efficacy or stability assessments. $(2,5,6,8,9,15,16)$ Moreover, the literature about studies conducted in the field lacks consistent data to enable comparing different treatment protocols and their stability.(5) The association between therapeutic protocol reports and randomized clinical trials (RCT) is essential to collect scientific evidences to improve the success and stability of AOB approaches. $(3,5,13)$

In light of the foregoing, the aims of the current randomized trial were to compare and evaluate the 2-year-follow-up stability of early $A O B$ treatment based on bonded lingual spurs (BS), chincup (CC), 
fixed palatal crib (FPC) and removable palatal crib (RPC). The null hypothesis was that all four treatment protocols would achieve similar short-term follow-up outcomes.

\section{Materials and methods}

The Ethics Committee of UNOPAR has approved the present investigation, which derived from a randomized study focused on comparing four experimental groups comprising AOB children treated with different devices.(14) The adopted methods were not subjected to any change after the experiments had begun, with the posttreatment follow-up planned from the study design stage.

Clinical study conducted with patients who were recruited based on the following inclusion criteria: children in the age group 7-10 years with Angle Class I relationship; AOB equal to, or larger than, 1.0 $\mathrm{mm}$; and fully erupted maxillary and mandibular permanent central incisors. $(10,11)$ All patients presented non-nutritive sucking and/or tongue thrusting habits. Information to characterize oral habits (type, duration, frequency and intensity) were collected through questionnaires applied to the children's parents and confirmed with a speech therapist. According the original study, $(14)$ the sample size was calculated based on an $\alpha$ of $5 \%$ and a power of $80 \%$ to detect an overbite mean difference of $1.75 \mathrm{~mm}$ among the groups with a standard deviation of $1.69 \mathrm{~mm}$. A total of 16 patients were needed in each group.

The initial sample comprised 99 patients who were divided, using a randon Excel spreadsheet (2007, Microsoft Windows) by a blind investigator, into four experimental groups: BS- bonded lingual spurs $(n=25) ; C C-$ chincup $(n=25) ;$ FPC- fixed palatal crib $(n=25)$ and; RPC- removable palatal crib $(n=24)$ (Figure 1).

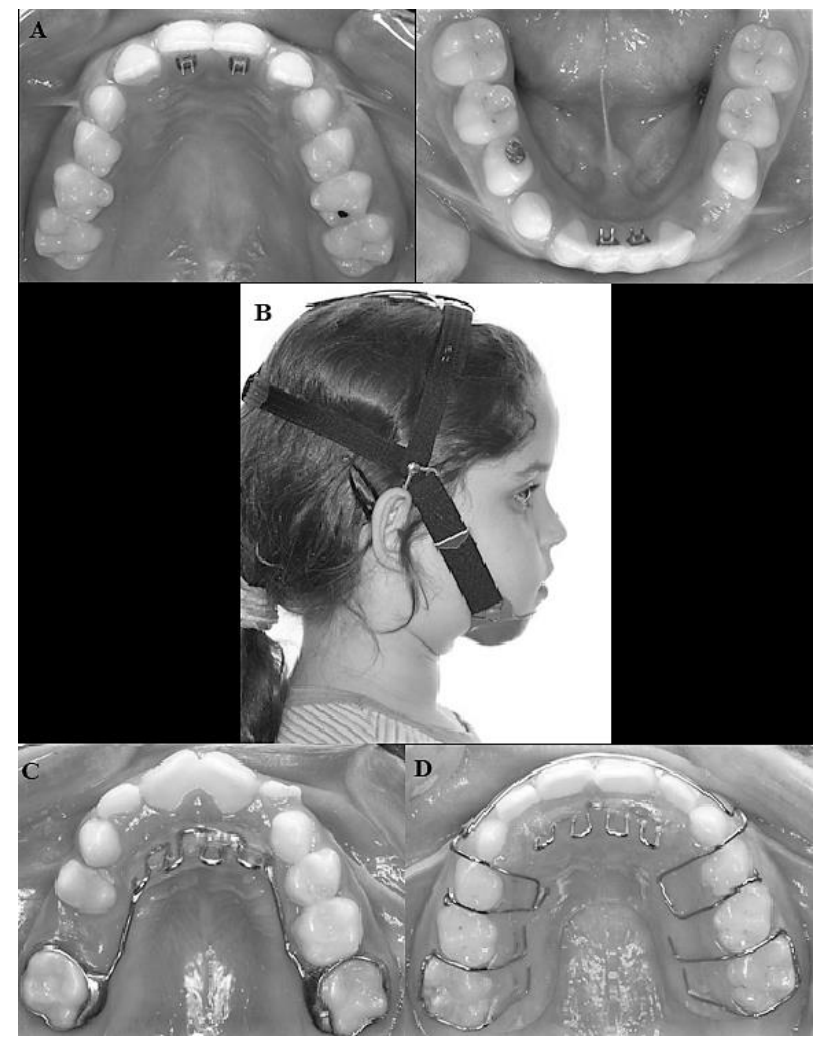

Figure 1. A. Bonded spurs (BS) in the maxillary and mandibular central incisors. B. Chincup (CC). C. Fixed palatal crib (FPC). D. Removable palatal crib (RPC).

The BS group was treated with Nogueira bonded lingual spurs (Abzil, 3M Unitek, São José do Rio Preto, SP, BR), which were bonded with Transbond $\mathrm{XT}^{\mathrm{TM}}$ orthodontic adhesive system (3M Unitek, Monrovia, Calif, USA). These devices were needle sharp with carborundum disc before installation (Figure $1 \mathrm{~A})$ by the same operator and fixed in the cervical and incisal portions of the maxillary and mandibular 
incisors, $(7,11,17)$ respectively, to prevent possible future occlusal interferences and to standardized their position and effects in all patients. The CC group was treated with night-time high-pull chincup (Morelli, Sorocaba, SP, BR) with force magnitude of approximately 450 to $500 \mathrm{~g}$ on each side(11) (Figure 1B).

The FPC group was treated with fixed crib, which included bands on the first permanent molars, added with palatal stainless-steel arch of $0.9 \mathrm{~mm}$ and palatal crib of stainless-steel wire of $0.7 \mathrm{~mm}$, throughout the length of the cervical lingual aspect of the mandibular incisors (Figure $1 \mathrm{C}$ ). Finally, the RPC group was treated with removable device composed of palatal crib, Adams' clasps on the permanent maxillary first molars, labial arch wire and acrylic coverage on the palatal area, which touched the palatal surface of all teeth, except for the maxillary incisors (Figure 1D). Patients were instructed to use the device full-time, except at meal and teeth brushing times. All children were examined monthly for positive reinforcement and the adjustment of appliances. Instructions were provided throughout the treatment to eliminate the sucking deleterious habits.

Eighty-four (84) patients (T2) have concluded their treatment after approximately one year. Sixtythree (63) patients who met inclusion criteria such as having participated in the previous AOB treatment and not having undergone any other orthodontic treatment or used retention device in the follow-up period were subjected to the 2-year-follow-up (T3) analysis.

Cephalograms of the initial treatment (T1), final treatment (T2) and 2-years-follow-up (T3) were traced by an investigator (F.A.D), based on customized digitization analysis, which was conducted in the Dolphin Imaging Software - Version 11.7 (Dolphin ${ }^{\circledR}$ Imaging and Management Solutions, Patterson Dental Supply, Inc., Chatsworth, CA). Skeletal, dental and soft tissue landmarks have generated cephalometric measurements; overbite was analyzed as the main outcome. $(4,9)$ Although the current research was a single-blind study when it came to cephalometric analysis, there was no blinding during the treatment due to the presence of adopted devices.

\section{Error study}

Thirty percent (30\%) of lateral cephalograms randomly selected from all observations were remeasured(18) to calculate the method error based on Dahlberg's formula. Systematic errors were evaluated through paired t-test, at $p<.05$. (19)

\section{Relapse index}

The relapse index of all variables ( $\Delta$ variable) at T3 was calculated as follow: $\Delta$ variable $=$ (T3valueT2value)//(T2value-T1 value)|. Negative values mean that measurements of T3 decreased in the follow up; on the other hand, positive values of this index mean that there was an increase in T3 measurements.

\section{Statistical analysis}

Statistical analysis was performed in the Statistica 7.0 software (StatSoft Inc., Tulsa, OK, USA), at $p<0.05$. Data distribution was analyzed by the Kolmogorov-Smirnov normality test, observing nonparametric data, described by median (Med), 25\% 75\%. Comparisons of variable relapses between the groups were performed by Kruskal-Wallis One Way Analysis of Variance on Ranks/Dunn post- hoc. Age comparison between experimental groups at T3 was carried out by one-way ANOVA and $\times 2$ test was used to compare intergroup sexual distribution and clinical success. Odds Ratio of deleterious oral habit breaking was calculated.

\section{Results}

Initially, cephalometric variables at pre-treatment (T1) have shown no significant intergroup difference (Table 1). When oral habits were analyzed through questionnaires applied before the treatments, the four experimental groups showed no significant difference related to type, duration, frequency and intensity. Patient compliance is critical to the success of clinical trials, but it is the hardest variables to control, resulting in drop-outs. The treatment results (T2-T1) were reported in previous study.(14) The final sample comprised 63 patients (Figure 2): BS ( $n=15$; mean overbite $0.19 \mathrm{~mm}$ and $11.54(0.63)$ years; 10 Female $(\mathrm{F}) / 5$ Male $(\mathrm{M}))$; $\mathrm{CC}(\mathrm{n}=11$; mean overbite $-0.19 \mathrm{~mm}$ and $11.41(0.89)$ years; $8 \mathrm{~F} / 3 \mathrm{M}) ; \mathrm{FPC}(\mathrm{n}=21$; mean overbite $1.23 \mathrm{~mm}$ and $11.44(0.93)$ years; $15 \mathrm{~F} / 6 \mathrm{M})$ and; $\mathrm{RPC}(\mathrm{n}=16$; mean overbite $0.73 \mathrm{~mm}$ and $11.67(1.11)$ years; $6 \mathrm{~F} / 10 \mathrm{M})$, with compatibility regarding age $(p=0.862)$ and sexual distribution $(p=0.136)$. 
Table 1. Intergroup comparison at T1 (baseline): Normality, Mean (M), Standard Deviation (SD); Analysis of Variance (p).

\begin{tabular}{|c|c|c|c|c|c|c|}
\hline Variables & Normality (p) & $\mathrm{BS}, \mathrm{n}=25$ & $C C, n=25$ & $\mathrm{FPC}, \mathrm{n}=25$ & $\mathrm{RPC}, \mathrm{n}=\mathbf{2 4}$ & $p$ \\
\hline \multicolumn{7}{|c|}{ Maxillary components } \\
\hline SNA $\left(^{\circ}\right)$ & 0.617 & $82.74 \pm 3.89$ & $83.86 \pm 2.71$ & $83.30 \pm 4.17$ & $82.65 \pm 3.31$ & 0.609 \\
\hline Co-A $(\mathrm{mm})$ & 0.145 & $76.44 \pm 3.80$ & $75.09 \pm 3.29$ & $75.10 \pm 3.94$ & $76.18 \pm 3.59$ & 0.430 \\
\hline \multicolumn{7}{|c|}{ Mandibular components } \\
\hline SNB $\left({ }^{\circ}\right)$ & 0.237 & $78.46 \pm 3.27$ & $78.78 \pm 3.17$ & $78.19 \pm 3.76$ & $77.10 \pm 2.75$ & 0.311 \\
\hline Co-Gn (mm) & 0.205 & $98.44 \pm 4.14$ & $98.55 \pm 4.50$ & $95.70 \pm 5.01$ & $96.89 \pm 4.66$ & 0.110 \\
\hline \multicolumn{7}{|c|}{ Maxillomandibular relationship } \\
\hline ANB $\left(^{\circ}\right)$ & 0.149 & $4.30 \pm 1.49$ & $5.09 \pm 2.11$ & $5.10 \pm 1.78$ & $5.54 \pm 2.02$ & 0.136 \\
\hline \multicolumn{7}{|l|}{ Vertical components } \\
\hline FMA $\left(^{\circ}\right)$ & 0.421 & $28.48 \pm 4.83$ & $30.06 \pm 4.46$ & $29.96 \pm 4.71$ & $29.15 \pm 3.80$ & 0.562 \\
\hline LAFH $(\mathrm{mm})$ & $0.013^{*}$ & $58.57 \pm 3.76$ & $59.26 \pm 4.04$ & $58.82 \pm 3.88$ & $58.43 \pm 4.44$ & 0.290 \\
\hline Overbite $(\mathrm{mm})$ & $<0.001^{*}$ & $-3.98 \pm 1.96$ & $-3.77 \pm 2.03$ & $-3.06 \pm 1.50$ & $-3.50 \pm 1.68$ & 0.201 \\
\hline \multicolumn{7}{|c|}{ Maxillary dentoalveolar componentes } \\
\hline U1.NA $\left(^{\circ}\right)$ & 0.385 & $29.27 \pm 6.01$ & $27.94 \pm 4.62$ & $25.77 \pm 4.49$ & $28.60 \pm 4.98$ & 0.089 \\
\hline U1.PP $\left({ }^{\circ}\right)$ & 0.368 & $117.58 \pm 6.56$ & $117.58 \pm 5.35$ & $114.80 \pm 5.49$ & $117.78 \pm 5.01$ & 0.195 \\
\hline U1-NA (mm) & 0.581 & $4.79 \pm 1.82$ & $4.42 \pm 1.45$ & $4.18 \pm \overline{1} .36$ & $4.11 \pm 1.47$ & 0.404 \\
\hline U1-PP (mm) & 0.732 & $21.98 \pm 2.12$ & $22.66 \pm 2.49$ & $23.14 \pm 2.47$ & $22.29 \pm 2.14$ & 0.330 \\
\hline U6-PP $(\mathrm{mm})$ & 0.474 & $16.50 \pm 1.99$ & $16.67 \pm 1.67$ & $16.52 \pm 1.70$ & $16.21 \pm 2.26$ & 0.866 \\
\hline \multicolumn{7}{|c|}{ Mandibular dentoalveolar componentes } \\
\hline $\operatorname{IMPA}\left({ }^{\circ}\right)$ & 0.452 & $94.40 \pm 6.31$ & $94.29 \pm 5.37$ & $93.22 \pm 6.89$ & $95.84 \pm 5.50$ & 0.514 \\
\hline L1.NB $\left(^{\circ}\right)$ & 0.263 & $30.46 \pm 5.09$ & $32.13 \pm 5.60$ & $30.15 \pm 6.42$ & $31.76 \pm 4.94$ & 0.522 \\
\hline L1-NB (mm) & 0.852 & $5.48 \pm 1.63$ & $6.40 \pm 1.86$ & $5.76 \pm 2.00$ & $5.91 \pm 1.67$ & 0.323 \\
\hline L1-MP (mm) & 0.329 & $33.52 \pm 2.22$ & $34.13 \pm 2.24$ & $33.83 \pm 2.54$ & $33.49 \pm 2.69$ & 0.771 \\
\hline L6-MP (mm) & 0.169 & $25.91 \pm 1.75$ & $26.17 \pm 1.81$ & $26.34 \pm 2.16$ & $26.10 \pm 1.83$ & 0.881 \\
\hline \multicolumn{7}{|c|}{ Soft tissue components } \\
\hline UL protrusion (mm) & 0.357 & $6.32 \pm 1.81$ & $6.62 \pm 1.69$ & $7.14 \pm 1.81$ & $6.94 \pm 1.17$ & 0.311 \\
\hline LL protrusion (mm) & 0.718 & $5.31 \pm 2.08$ & $5.99 \pm 1.76$ & $6.04 \pm 2.56$ & $5.93 \pm 1.89$ & 0.580 \\
\hline Nasolabial Angle $\left({ }^{\circ}\right)$ & 0.846 & $104.63 \pm 7.54$ & $104.28 \pm 8.33$ & $103.85 \pm 7.59$ & $105.27 \pm 7.23$ & 0.930 \\
\hline
\end{tabular}




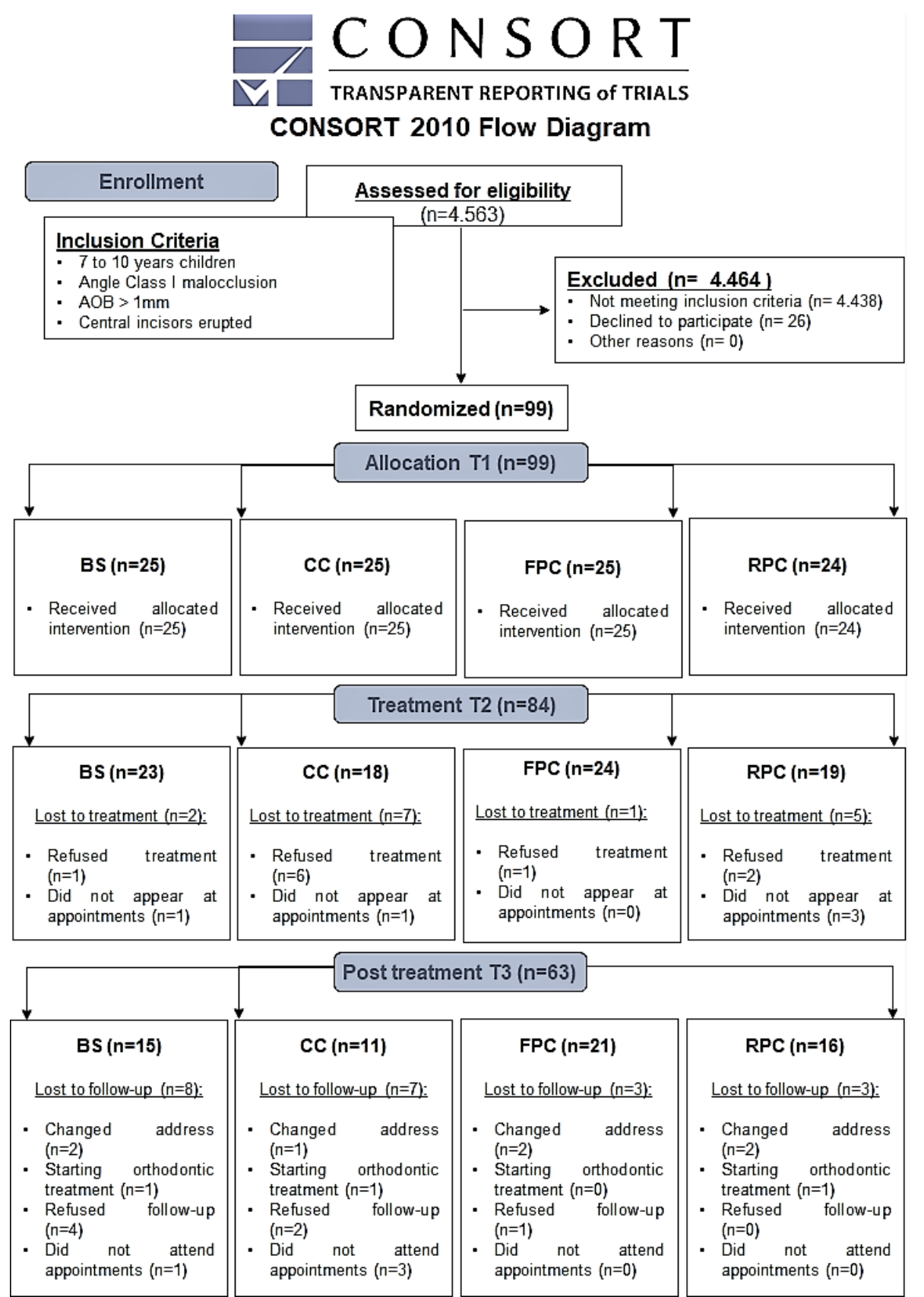

Figure 2. Diagram of Consolidated Standards of Reporting Trials (CONSORT) showing patients' flow through the trial.

The angular maxillary and mandibular components (SNA; SNB; ANB) did not significantly change throughout the study. On the other hand, linear component ( $\left.\mathrm{Co}_{-} \mathrm{A}_{i} \mathrm{Co}-\mathrm{Gn}\right)$ measurements have increased. The maxillary component has gradually changed overtime whereas the mandibular one has shown difference at pubertal growth spurt (between T2 and T3), except for the CC group, whose patients presented smaller mandibular change between T2 and T3 (Table 2). 
Table 2. Intergroup comparisons of changes occurred in 2-years-post-treatment ( $\Delta$ Variable=T3-T2/|T2-T1|): Median (Med), 25\% 75\%; Kruskal-Wallis One Way Analysis of Variance on Ranks/Dunn post- hoc

\begin{tabular}{|c|c|c|c|c|c|}
\hline Variables & $\begin{array}{c}\text { BS, } n=15 \\
\text { Med } \\
(25 \% / 75 \%)\end{array}$ & $\begin{array}{c}\text { CC, } n=11 \\
\text { Med } \\
(25 \% / 75 \%)\end{array}$ & $\begin{array}{c}\text { FPC, } n=21 \\
\text { Med } \\
(25 \% / 75 \%)\end{array}$ & $\begin{array}{c}\text { RPC, } n=16 \\
\text { Med } \\
(25 \% / 75 \%)\end{array}$ & $p$ \\
\hline \multicolumn{6}{|c|}{ Maxillary components } \\
\hline SNA $\left(^{\circ}\right)$ & $\begin{array}{c}-0.33 \\
(-0.66 / 1.48)\end{array}$ & $\begin{array}{c}0.44 \\
(0.00 / 0.86)\end{array}$ & $\begin{array}{c}-0.33 \\
(-1.44 / 0.53)\end{array}$ & $\begin{array}{c}0.80 \\
(-1.07 / 3.12)\end{array}$ & 0.431 \\
\hline Co-A (mm) & $\begin{array}{c}2.14 \\
(0.89 / 5.62)\end{array}$ & $\begin{array}{c}1.17 \\
(0.16 / 6.67)\end{array}$ & $\begin{array}{c}2.13 \\
(0.87 / 2.77)\end{array}$ & $\begin{array}{c}1.58 \\
(0.05 / 5.34)\end{array}$ & 0.966 \\
\hline \multicolumn{6}{|c|}{ Mandibular components } \\
\hline SNB $\left({ }^{\circ}\right)$ & $\begin{array}{c}1.07 \\
(-0.04 / 3.00)\end{array}$ & $\begin{array}{c}1.00 \\
(0.17 / 2.00)\end{array}$ & $\begin{array}{c}0.51 \\
(-1.29 / 3.70)\end{array}$ & $\begin{array}{c}1.79 \\
(-0.36 / 2.50)\end{array}$ & 0.837 \\
\hline Co-Gn (mm) & $\begin{array}{c}2.64 \\
(0.88 / 5.09)\end{array}$ & $\begin{array}{c}1.52 \\
(0.42 / 3.54)\end{array}$ & $\begin{array}{c}2.73 \\
(1.18 / 5.80)\end{array}$ & $\begin{array}{c}1.36 \\
(0.72 / 2.74)\end{array}$ & 0.279 \\
\hline \multicolumn{6}{|c|}{ Maxillomandibular relationship } \\
\hline ANB $\left(^{\circ}\right)$ & $\begin{array}{c}-0.68 \\
(-4.50 / 0.09)\end{array}$ & $\begin{array}{c}-0.67 \\
(-2.29 / 0.37)\end{array}$ & $\begin{array}{c}-1.45 \\
(-6.37 /-0.07)\end{array}$ & $\begin{array}{c}-1.08 \\
(-3.90 / 0.19)\end{array}$ & 0.772 \\
\hline \multicolumn{6}{|l|}{ Vertical components } \\
\hline FMA $\left(^{\circ}\right)$ & $\begin{array}{c}-0.83 \\
(-2.00 / 0.14)\end{array}$ & $\begin{array}{c}-1.33 \\
(-4.33 / 0.48)\end{array}$ & $\begin{array}{c}-0.59 \\
(-1.52 / 0.29)\end{array}$ & $\begin{array}{c}0.47 \\
(-0.36 / 1.15)\end{array}$ & 0.151 \\
\hline LAFH (mm) & $\begin{array}{c}2.90 \\
(1.00 / 13.00)\end{array}$ & $\begin{array}{c}0.87 \\
(-0.12 / 1.28)\end{array}$ & $\begin{array}{c}1.23 \\
(0.50 / 3.54)\end{array}$ & $\begin{array}{c}0.60 \\
(-0.93 / 2.74)\end{array}$ & 0.078 \\
\hline Overbite (mm) & $\begin{array}{c}0.46 \\
(0.00 / 1.69)\end{array}$ & $\begin{array}{c}1.10 \\
(0.11 / 1.60)\end{array}$ & $\begin{array}{c}0.40 \\
(-0.12 / 0.89)\end{array}$ & $\begin{array}{c}0.15 \\
(-0.06 / 0.67)\end{array}$ & 0.261 \\
\hline \multicolumn{6}{|c|}{ Maxillary dentoalveolar componentes } \\
\hline U1.NA $\left({ }^{\circ}\right)$ & $\begin{array}{c}-0.18^{a, b} \\
(-0.95 / 0.099)\end{array}$ & $\begin{array}{c}-1.14^{\mathrm{a}} \\
(-4.00 /-0.22)\end{array}$ & $\begin{array}{c}0.43^{b} \\
(-0.12 / 2.22)\end{array}$ & $\begin{array}{c}0.63^{b} \\
(-0.37 / 0.94)\end{array}$ & $0.005^{*}$ \\
\hline U1.PP $\left({ }^{\circ}\right)$ & $\begin{array}{c}-0.20 \\
(-0.94 / 1.47)\end{array}$ & $\begin{array}{c}-0.81 \\
(-2.20 / 0.33)\end{array}$ & $\begin{array}{c}0.31 \\
(-0.31 / 0.97)\end{array}$ & $\begin{array}{c}0.60 \\
(0.21 / 1.06)\end{array}$ & 0.085 \\
\hline U1-NA (mm) & $\begin{array}{c}0.71^{a, b} \\
(-0.46 / 1.86)\end{array}$ & $\begin{array}{c}-0.57^{\mathrm{a}} \\
(-3.75 / 1.33)\end{array}$ & $\begin{array}{c}1.18^{b} \\
(0.27 / 2.00)\end{array}$ & $\begin{array}{c}1.84^{b} \\
(1.11 / 7.25)\end{array}$ & $0.008^{*}$ \\
\hline U1-PP (mm) & $\begin{array}{c}1.30^{\mathrm{a}, \mathrm{b}} \\
(0.50 / 1.68)\end{array}$ & $\begin{array}{c}1.92^{\mathrm{a}} \\
(1.31 / 3.00)\end{array}$ & $\begin{array}{c}0.97^{\mathrm{a}} \\
(0.61 / 2.08)\end{array}$ & $\begin{array}{c}0.54^{\mathrm{b}} \\
(0.03 / 0.74)\end{array}$ & $<0.001^{*}$ \\
\hline U6-PP (mm) & $\begin{array}{c}3.40 \\
(1.38 / 8.33)\end{array}$ & $\begin{array}{c}0.00 \\
(-0.60 / 7.67)\end{array}$ & $\begin{array}{c}1.41 \\
(0.30 / 2.61)\end{array}$ & $\begin{array}{c}2.03 \\
(0.10 / 7.25)\end{array}$ & 0.068 \\
\hline \multicolumn{6}{|c|}{ Mandibular dentoalveolar componentes } \\
\hline $\operatorname{IMPA}\left({ }^{\circ}\right)$ & $\begin{array}{c}-0.02 \\
(-0.95 / 1.34)\end{array}$ & $\begin{array}{c}-0.27 \\
(-0.66 / 0.56)\end{array}$ & $\begin{array}{c}0.85 \\
(0.31 / 1.51)\end{array}$ & $\begin{array}{c}0.34 \\
(-5.41 / 1.63)\end{array}$ & 0.063 \\
\hline L1.NB $\left({ }^{\circ}\right)$ & $\begin{array}{c}0.06 \\
(-0.74 / 1.75)\end{array}$ & $\begin{array}{c}-0.21 \\
(-1.05 / 0.28)\end{array}$ & $\begin{array}{c}0.66 \\
(0.32 / 1.36)\end{array}$ & $\begin{array}{c}0.48 \\
(-2.07 / 0.99)\end{array}$ & 0.129 \\
\hline L1-NB (mm) & $\begin{array}{c}1.67 \\
(0.07 / 3.33)\end{array}$ & $\begin{array}{c}0.22 \\
(-1.00 / 0.92)\end{array}$ & $\begin{array}{c}1.77 \\
(0.78 / 4.50)\end{array}$ & $\begin{array}{c}0.85 \\
(-0.64 / 1.63)\end{array}$ & 0.022 \\
\hline L1-MP (mm) & $\begin{array}{c}1.25 \\
(0.50 / 2.50)\end{array}$ & $\begin{array}{c}0.52 \\
(-0.08 / 2.27)\end{array}$ & $\begin{array}{c}0.90 \\
(0.35 / 1.78)\end{array}$ & $\begin{array}{c}0.54 \\
(0.31 / 1.55)\end{array}$ & 0.741 \\
\hline L6-MP (mm) & $\begin{array}{c}0.47 \\
(-0.22 / 1.33)\end{array}$ & $\begin{array}{c}2.67 \\
(-0.75 / 3.67)\end{array}$ & $\begin{array}{c}0.78 \\
(0.01 / 2.52)\end{array}$ & $\begin{array}{c}0.61 \\
(-0.86 / 1.93)\end{array}$ & 0.574 \\
\hline \multicolumn{6}{|c|}{ Soft tissue components } \\
\hline UL protrusion (mm) & $\begin{array}{c}0.50 \\
(-1.00 / 1.60)\end{array}$ & $\begin{array}{c}-2.01 \\
(-5.00 / 0.33)\end{array}$ & $\begin{array}{c}-1.00 \\
(-2.67 /-0.06)\end{array}$ & $\begin{array}{c}-0.01 \\
(-4.17 / 0.48)\end{array}$ & 0.259 \\
\hline LL protrusion $(\mathrm{mm})$ & $\begin{array}{c}-0.30 \\
(-2.68 / 1.03)\end{array}$ & $\begin{array}{c}-0.62 \\
(-3.14 / 0.92)\end{array}$ & $\begin{array}{c}0.39 \\
(-1.37 / 0.93)\end{array}$ & $\begin{array}{c}-0.49 \\
(-1.89 / 0.66)\end{array}$ & 0.600 \\
\hline Nasolabial Angle $\left({ }^{\circ}\right)$ & $\begin{array}{c}-0.71 \\
(-2.80 / 1.00)\end{array}$ & $\begin{array}{c}0.25 \\
(-1.23 / 1.05)\end{array}$ & $\begin{array}{c}-0.26 \\
(-1.03 / 3.77)\end{array}$ & $\begin{array}{c}0.53 \\
(-0.54 / 3.78)\end{array}$ & 0.227 \\
\hline
\end{tabular}

BS indicate bonded spurs; CC, chin cup; FPC, fixed palatal crib; RPC, removable palatal crib; LAFH, lower anterior facial height; UL, upper lip; LL, lower lip; Nasolabial Angle, Cm.Sn.UL.

* Statistically significant difference / Different letters indicate statistically difference. 
Changes in dentoalveolar component measurements presented statistically significant differences between the groups. Angular components tended to decrease during treatment (T2-T1), resulting in more vertical anterior teeth; meanwhile at post-treatment, there were relapses significantly higher in FPC and RPC groups. Regarding the linear dentoalveolar components, there was extrusion of incisors during the treatment period, without relapses and differences between groups at T3. Soft tissue components did not show significant changes (Table 2).

As the main outcome in this analysis, the overbite has improved in all groups throughout the treatment (T2-T1) and in the post-treatment period (Figure 3), with no statistical difference between the four groups. The mean improvement of overbite was $4.09 \pm 2.15 \mathrm{~mm}$ in this study, with $1.15 \mathrm{~mm}$ in the post-treatment (T3-T2) (Figure 3 and 4). The differences in the median overbite values among the treatment groups were not great enough to exclude the possibility that the difference was due to random sampling variability; but there was not a statistically significant difference $(p=0.261)$.

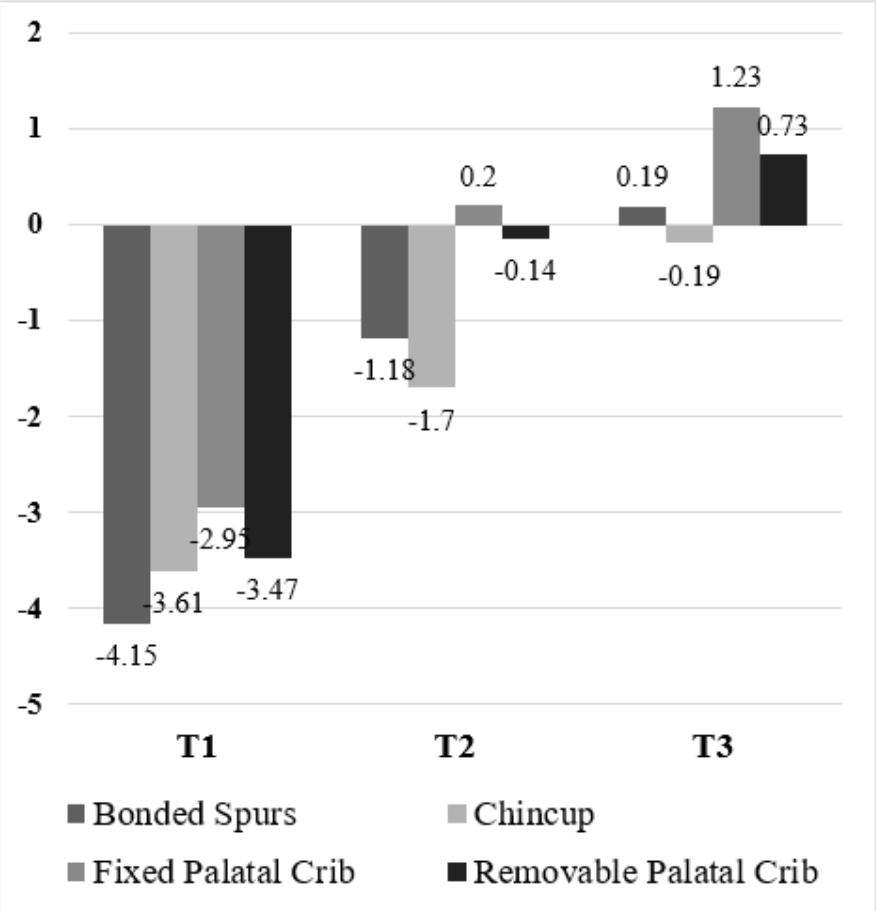

Figure 3. Overbite behavior throughout the study.

T1

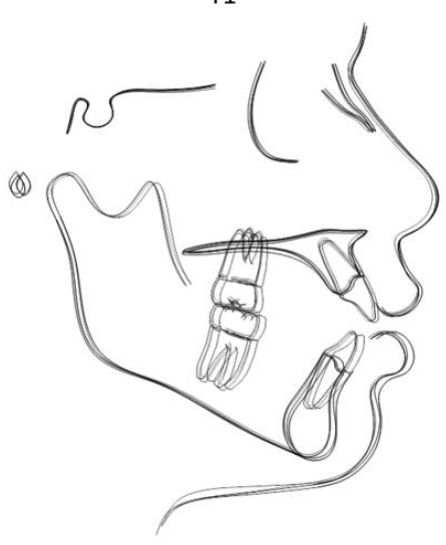

T2

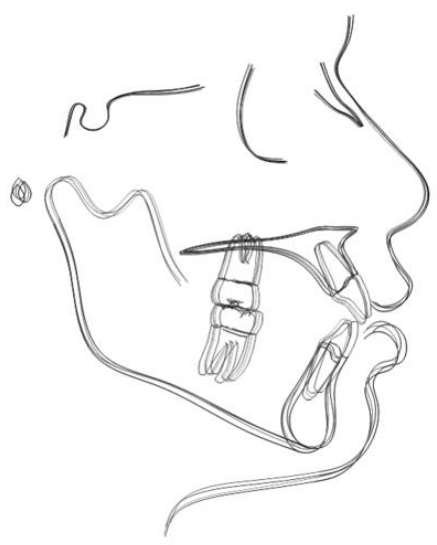

T3

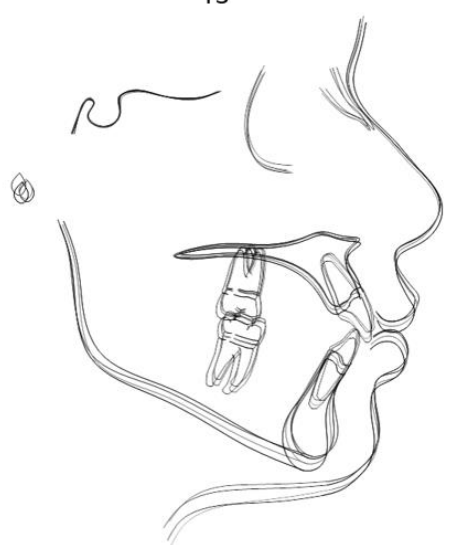

Figure 4. Mean superimposition of initial (T1), final (T2) and 2-year post-treatment (T3) cephalometric tracings of 4 experimental groups, based on the SN plane, showing the overbite improvement throughout the study. 
Three patients presenting positive measurements in $T 2$, had clinically significant relapse in $T 3$, presenting negative overbite $(4,9)(\mathrm{FPC}=2 ; \mathrm{RPC}=1 ; 4.76 \%$ of total sample). Forty-six patients $(\mathrm{BS}=12$; $\mathrm{CC}=9 ; \mathrm{FPC}=14 ; \mathrm{RPC}=11 ; 73.02 \%$ ) presented overbite measurement stability in the post-treatment period (overbite T3-T2). The remaining fourteen patients got to the end of the study without achieving overbite measurement stability (negative changes); however, this circumstance did not change previous treatment results. The greatest clinical success was observed in the FPC group at T3 (80.95\%).

After treatment, 70.97\% of patients had broken deleterious oral habits; patients who used FPC $(90.48 \%)$ and RPC (81.25\%) recorded higher deleterious oral habit breaking rates than the ones using BS (53.33\%) and CC (45.45\%). Patients who abandoned deleterious oral habits recorded $54.44 \%$ relative increase in AOB treatment success, as well as 25.5 times more likelihood of vertical trespass correction, at significance level of $p<0.05$.

\section{Discussion}

Several devices have been investigated for the early treatment of $A O B ;(7,10,11,14,20-22)$ however, results of stability and long-term follow-up remains under discussion due the limited data available $(5,23)$ showing some follow-up periods along with complete fixed appliance therapy. $(3,15)$ The present study evaluated changes observed in the post-treatment period of patients subjected to four different devices $(B S, C C, F P C, R P C)$, with emphasis on the isolated effects of each treatment, without the use of any other orthodontic fixed therapy after proposed early treatments. It is essential assessing the follow-up stability after orthodontic treatments due to risk of relapse.(6) The 2-year follow-up (T3) was considered adequate for stability investigations, since significant relapse can take place during this period.(24)

Patient compliance is critical to the success of clinical trials and it becomes evident in stability studies. The reasons for drop-outs in this study were inability to contact the patient due changes in address or telephone's number, orthodontic treatment started by other professionals, refusal to continue participating in the research and not attended appointments. These inevitable losses resulted in low power test, below the desired of 0.8. However, this study was applied to patients with similar characteristics, minimizing the bias. Thus, the results should be interpreted cautiously; therefore, further well-conducted trials with long-term post treatment follow-up assessments are still necessary, creating more evidence to support the results of early treatment of the AOB.

The lack of untreated control group can be justified by attention of the sample age as ideal to correct the investigated malocclusion.(1) Children follow-up for approximately 3 years would be ethically unacceptable. Since all groups were compatible at the beginning of the study, changes associated with normal growth have presented similar behavior.(25) This outcome has shown that adequate samples were allocated to equal groups, and it enabled a true RCT.(23)

The comparison between initial measurement changes and values recorded at the 2-year follow-up was favorable to the success of early AOB treatment.(23) The improved vertical relationship with no statistically significant differences in overbite observed in the intergroup has mainly resulted from dentoalveolar effects - it did not significantly change skeletal components. It may have happened due to short treatment time, a fact that has corroborated previous studies. $(10,11)$ Anterior teeth extrusion (greater in the FPC group) was an important factor favoring AOB correction and stability; besides, it corroborated findings by Mucedero et al,(15) who determined that extrusion was a critical factor for AOB correction stability. As overbite was the main research outcome, the null hypothesis was confirmed.

Tipping improvement, in association with the extrusion of maxillary and mandibular dentoalveolar components during treatment, may due to decreased or blocked tongue thrusting, anterior tongue resting posture and sucking habits enabled by the adopted devices, $(7,10,21,26-28)$ mainly by FPC and $\operatorname{RPC} .(8,12,14,22)$

The incidence of deleterious oral habits at the mixed dentition phase is the main etiological factor of AOB.(1) Devices adopted in the current study appeared to be effective in helping children abandon deleterious oral habits - sucking cessation index in the total sample reached approximately $71 \%$. This outcome supports the conclusion by Borrie et al(26) and Feres et al,(27) according to whom habitinterception devices enabled a larger number of children to abandon sucking habits than no-treatment groups. The identification of negative overbite in some patients at the end of treatment (T2) can be partly associated with non-abandonment of deleterious oral habits, since the likelihood of overbite correction was 25.5 times higher in patients who had abandoned them.

The FPC device presented clinical advantages such as lower patient withdrawal (84\% survival rate in T3) and AOB correction at T3 (80. 95\%). The observed changes were associated with tongue posture 
adjustment and with the early management of deleterious oral habits. $(7,27)$ Previous studies $(7,21,28)$ have already shown the effectiveness of therapies based on palatal cribs to discontinue sucking habits. The selection of the best AOB interception device to be applied at patients' early age should be based on malocclusion etiology and features, as well as on patients' cooperation.(7) RPC may be a proper choice when maxillary incisors' inclination and position are the most prominent change, whereas FPC has better response if changes in mandibular incisors are more evident. Based on patients' cooperation, the best results - such as lesser withdrawal and greater cessation of deleterious oral habits - were observed in the FPC group.

Significantly greater relapse in maxillary and mandibular incisors' inclination and position was observed during stability period in the FPC and RPC groups. These were exactly the two groups presenting greater treatment-related changes. This effect may have been caused by device removal and by tongue action on patients' incisors, $(4,8,9)$ which would indicate that the cribs did not retrain, but only restrained, patients' tongue - a process that would establish a new posture due to nociceptive or proprioceptive reflex.(17)

Despite the relapses, cephalometric measurements tended to follow dentofacial growth and development. It is possible assuming that changes observed in the BS and CC groups reflected growth changes expected at this phase, such as the muscular balance achieved with the late cessation of deleterious oral habits.

The results of the present trial showed favorable changes related overbite during the post treatment phase,(29) with a clinically significant relapse rate of $4.76 \%$ that was similar to that $4 \%$ recorded by Cassis et al(9) and $5.3 \%$ by Ferreira et al(8) in the post-treatment period of patients subjected to $\mathrm{BS} / \mathrm{CC}$ and $\mathrm{RPC} / \mathrm{CC}$, respectively. This outcome has shown that the early AOB treatment achieved notable stability results.

In conclusion, incisors' tipping tended to relapse in the FPC and RPC groups because the crib did not retrain the tongue, it only restrained it. Incisors' extrusion has influenced AOB correction and stability in all four groups. For the reasons aforementioned, within limitations in this study, all appliances used were effective and showed stable results in the early AOB treatment, being FPC the device recorded the highest $A O B$ correction and the lowest patient withdrawal rate.

\section{Resumo}

Os objetivos do presente estudo foram comparar e avaliar a estabilidade do tratamento precoce da mordida aberta anterior (MAA) com diferentes dispositivos. A amostra inicial foi composta por 99 pacientes randomizados em quatro grupos experimentais: BS - esporões colados; CC - mentoneira; FPC - grade palatina fixa; RPC - grade palatina removivel. Análise cefalométrica foi realizada para avaliar os dados do período inicial (T1), final do tratamento (T2) e 2 anos após tratamento (T3), sendo a variável overbite o desfecho principal. Em T3, após perdas de seguimento, haviam 63 individuos, sendo BS $(n=15$; overbite $0.19 \mathrm{~mm} ; 11.54$ anos; 10 Feminino (F)/5 Masculino (M)); CC ( $\mathrm{n}=11$; overbite $-0.19 \mathrm{~mm} ; 11.41$ anos; $8 \mathrm{~F} / 3 \mathrm{M})$; FPC ( $\mathrm{n}=21$; overbite $1.23 \mathrm{~mm} ; 11.44$ anos; $15 \mathrm{~F} / 6 \mathrm{M})$ e; RPC $(\mathrm{n}=16$; overbite $0.73 \mathrm{~mm}$; 11.67 anos; $6 \mathrm{~F} / 10 \mathrm{M}$ ). Comparações das alterações nas variáveis dentoesqueléticas e abandono de hábitos bucais deletérios durante o tempo de acompanhamento foram estatisticamente analisados com $p<.05$. Medidas esqueléticas lineares mandibulares e componentes verticais aumentaram gradualmente com a idade, principalmente com o surto de crescimento puberal e estabelecimento da dentição permanente no pós-tratamento. 0 overbite foi significantemente melhorado durante o tratamento, permanecendo estável com alterações positivas. A extrusão dos incisivos impactou na correção da MAA e estabilidade nos 4 grupos, que registraram uma melhora de $1.15 \mathrm{~mm}$ no pós-tratamento (T3-T2). Com suas limitações, todos dispositivos experimentais foram efetivos e mostraram resultados estáveis no tratamento precoce da MAA, sendo que a FPC apresentou a maior correção da MAA e o menor índice de desistência. 


\section{References}

1. Almeida RR, Almeida-Pedrin RR, Almeida MR, Ferreira FPC, Pinzan A, Insabralde CMB. Vertical Dysplasias: Anterior Open Bite - Treatment and Stability. R Dental Press Ortodon Ortop Facial 2003;8(4):91-119.

2. Lopez-Gavito G, Wallen TR, Little RM, Joondeph DR. Anterior open-bite malocclusion: a longitudinal 10-year postretention evaluation of orthodontically treated patients. Am J Orthod 1985;87(3):175-186.

3. Cozza P, Mucedero M, Baccetti T, Franchi L. Early orthodontic treatment of skeletal open-bite malocclusion: a systematic review. Angle Orthod 2005;75(5):707-713.

4. Janson G, Valarelli FP, Beltrao RT, de Freitas MR, Henriques JF. Stability of anterior open-bite extraction and nonextraction treatment in the permanent dentition. Am J Orthod Dentofacial Orthop 2006;129(6):768-774.

5. Greenlee GM, Huang GJ, Chen SS, Chen J, Koepsell T, Hujoel P. Stability of treatment for anterior open-bite malocclusion: a meta-analysis. Am J Orthod Dentofacial Orthop 2011;139(2):154-169.

6. Bondemark L, Holm AK, Hansen K, Axelsson S, Mohlin B, Brattstrom Vet al. Long-term stability of orthodontic treatment and patient satisfaction. A systematic review. Angle Orthod 2007;77(1):181-191.

7. Parker JH. The interception of the open bite in the early growth period. Angle Orthod 1971;41(1):24-44.

8. Ferreira FPC, Almeida RR, Torres PC, Almeida-Pedrin RR, Almeida MR, Santana Filho R. Evaluation of the stability of open bite treatment using a removable appliance with palatal crib combined with high-pull chincup. Dental Press J Orthod 2012;17(6):52-60.

9. Cassis MA, de Almeida RR, Janson G, Aliaga-Del Castillo A, de Almeida MR. Stability of anterior open bite treatment with bonded spurs associated with high-pull chincup. Orthod Craniofac Res 2018;21(2):104-111.

10. Canuto LF, Janson G, de Lima NS, de Almeida RR, Cancado RH. Anterior open-bite treatment with bonded vs conventional lingual spurs: A comparative study. Am J Orthod Dentofacial Orthop 2016;149(6):847-855.

11. Cassis MA, de Almeida RR, Janson $G$, de Almeida-Pedrin RR, de Almeida MR. Treatment effects of bonded spurs associated with high-pull chincup therapy in the treatment of patients with anterior open bite. Am J Orthod Dentofacial Orthop 2012;142(4):487-493.

12. Insabralde NM, de Almeida RR, Henriques JF, Fernandes TM, Flores-Mir C, de Almeida MR. Dentoskeletal effects produced by removable palatal crib, bonded spurs, and chincup therapy in growing children with anterior open bite. Angle Orthod 2016;86(6):969-975.

13. Lentini-Oliveira DA, Carvalho FR, Rodrigues CG, Ye O, Prado LB, Prado GFet al. Orthodontic and orthopaedic treatment for anterior open bite in children. Cochrane Database Syst Rev 2014(9):CD005515.

14. Rossato PH, Fernandes TMF, Urnau FDA, de Castro AC, Conti F, de Almeida RRet al. Dentoalveolar effects produced by different appliances on early treatment of anterior open bite: A randomized clinical trial. Angle Orthod 2018;88(6):684-691.

15. Mucedero M, Franchi L, Giuntini V, Vangelisti A, McNamara JA, Jr., Cozza P. Stability of quadhelix/crib therapy in dentoskeletal open bite: a long-term controlled study. Am J Orthod Dentofacial Orthop 2013;143(5):695-703.

16. Teittinen M, Tuovinen V, Tammela L, Schatzle M, Peltomaki T. Long-term stability of anterior open bite closure corrected by surgical-orthodontic treatment. Eur J Orthod 2012;34(2):238-243.

17. Justus R. Correction of anterior open bite with spurs: long-term stability. World J Orthod $2001 ; 2(3): 219-231$.

18. Houston WJ. The analysis of errors in orthodontic measurements. Am J Orthod 1983;83(5):382390.

19. Cancado RH, Lauris JR. Error of the method: what is it for? Dental Press J Orthod 2014;19(2):2526.

20. Iscan HN, Dincer M, Gultan A, Meral O, Taner-Sarisoy L. Effects of vertical chincap therapy on the mandibular morphology in open-bite patients. Am J Orthod Dentofacial Orthop 2002;122(5):506511.

21. Leite JS, Matiussi LB, Salem AC, Provenzano MG, Ramos AL. Effects of palatal crib and bonded spurs in early treatment of anterior open bite: A prospective randomized clinical study. Angle Orthod 2016;86(5):734-739. 
22. Slaviero T, Fernandes TM, Oltramari-Navarro PV, de Castro AC, Conti $F$, Poleti MLet al. Dimensional changes of dental arches produced by fixed and removable palatal cribs: A prospective, randomized, controlled study. Angle Orthod 2017;87(2):215-222.

23. Feres MF, Abreu LG, Insabralde NM, Almeida MR, Flores-Mir C. Effectiveness of the open bite treatment in growing children and adolescents. A systematic review. Eur J Orthod 2016;38(3):237-250.

24. Al Yami EA, Kuijpers-Jagtman AM, van 't Hof MA. Stability of orthodontic treatment outcome: follow-up until 10 years postretention. Am J Orthod Dentofacial Orthop 1999;115(3):300-304.

25. Phelan A, Franchi L, Baccetti T, Darendeliler MA, McNamara JA, Jr. Longitudinal growth changes in subjects with open-bite tendency: a retrospective study. Am J Orthod Dentofacial Orthop 2014;145(1):28-35.

26. Borrie FR, Bearn DR, Innes NP, Iheozor-Ejiofor Z. Interventions for the cessation of non-nutritive sucking habits in children. Cochrane Database Syst Rev 2015(3):CD008694.

27. Feres MF, Abreu LG, Insabralde NM, de Almeida MR, Flores-Mir C. Effectiveness of open bite correction when managing deleterious oral habits in growing children and adolescents: a systematic review and meta-analysis. Eur J Orthod 2017;39(1):31-42.

28. Huang GJ, Justus R, Kennedy DB, Kokich VG. Stability of anterior openbite treated with crib therapy. Angle Orthod 1990;60(1):17-24; discussion 25-16.

29. Riolo ML, University of M, Center for Human G, Development. An atlas of craniofacial growth : cephalometric standards from the University School growth study, the University of Michigan by Michael L. Riolo ... [et al.]. Ann Arbor, Mich.: Center for Human Growth and Development, the University of Michiagan; 1979. 